\title{
Cannabis, Cannabinoide und Cannabiskonsumstörungen
}

Eva Hoch, Ulrich W. Preuss

\begin{abstract}
Keine Droge führt derzeit zu so intensiven, teilweise leidenschaftlich geführten Diskussionen wie Cannabis. Welche gesundheitlichen und sozialen Risiken birgt die Substanz, wenn sie zu Rauschzwecken gebraucht wird? Dieser Beitrag beschreibt die wichtigsten Cannabinoide, das endogene Cannabissystem sowie mögliche kurzund langfristige Risiken. Er zeigt zudem psychotherapeutische Behandlungsoptionen von Cannabismissbrauch und -abhängigkeit auf.
\end{abstract}

\section{Einleitung}

Etwa 24,7 Millionen Bürger der Europäischen Union konsumieren Cannabis zu Rauschzwecken, ca. 3 Millionen (1\%) davon klinisch relevant. Wie ein Mensch auf Cannabis reagiert, ist individuell unterschiedlich. Wissenschaftlich belegt sind erhöhte Risiken für kognitive Störungen, Einbußen im Bildungserfolg, Psychosen, Depressionen, bipolare Störungen, Angsterkrankungen sowie psychische und körperliche Abhängigkeit im Zusammenhang mit Cannabis. Faktoren wie Alter bei Erstkonsum, Dauer, Menge und Art des konsumierten Cannabis sowie eine individuelle Vulnerabilität spielen dabei eine Rolle.

\section{Merke \\ Cannabis ist nach Alkohol der häufigste Anlass für eine Suchtbehandlung in Deutschland.}

Das cannabisspezifische Entzugssyndrom lässt sich klinisch gut behandeln. Psychotherapeutische Interventionen beinhalten Kombinationen aus Motivationsförderung und kognitiver Verhaltenstherapie. Bei Kindern und Jugendlichen können familientherapeutische Ansätze hilfreich sein. Die Erfolgsraten der psychotherapeutischen Behandlungsansätze sind moderat, die Rückfallraten hoch. Effektive medikamentöse Optionen fehlen. Aus diesem Grund kommt der Prävention von riskantem, schädlichem und abhängigem Cannabisgebrauch eine tragende gesundheitspolitische Rolle zu.

\section{Cannabis - eine Alltagsdroge?}

Cannabis zählt zusammen mit Alkohol und Tabak zu den am häufigsten gebrauchten psychotropen Substanzen weltweit. Die häufigsten 2 Konsumformen von pflanzlichem Cannabis sind:
- die getrockneten Blüten und Blätter der weiblichen Hanfpflanze (Marihuana) und

- das aus dem THC-( $\Delta$ 9-Tetrahydrocannabinol-)haltigen Harz der Blütenstände gewonnene, meist zu bräunlich-grünen Platten gepresste Haschisch.

Seltener nehmen die Konsumenten THC-haltiges Öl in Nahrungsmitteln zu sich [1].

\section{Konsumprävalenz}

Laut aktuellstem Weltdrogenbericht haben 4,3\% der erwachsenen Bevölkerung im letzten Jahr Cannabis gebraucht [2]. Das entspricht rund 192,2 Millionen Menschen. Für Europa gehen die jüngsten Schätzungen davon aus, dass 6,6\% der Bürger der Europäischen Union im Alter von 15 bis 64 Jahren Cannabis in den letzten 12 Monaten gebraucht haben (rund 24,7 Millionen) [3]. In Deutschland wird die 12-Monats-Prävalenz des Cannabiskonsums bei 18- bis 64-jährigen in der Allgemeinbevölkerung auf $6,1 \%$ geschätzt, dies entspricht rund 3,11 Millionen Cannabiskonsumenten [4]. Deutlich höhere Konsumprävalenzen wurden bei jungen Erwachsenen beobachtet. So berichtet eine Jugendstudie von einer 12-Monatsprävalenz von $17,6 \%$ bei 18 - bis 25 -Jährigen für das Jahr 2014 [5]. In dieser Altersgruppe konsumierten mehr Männer (22,1\%) Cannabis als Frauen (12,9\%).

Diese Zahlen stammen aus epidemiologischen Bevölkerungsstudien und sind ein Anhaltspunkt dafür, in welcher Größenordnung Cannabis gebraucht wurde. Sie geben keine Auskunft darüber, um welche Art des Konsums es sich dabei handelt (z. B. um einen einmaligen, regelmäßigen, riskanten, problematischen, missbräuchlichen oder abhängigen Gebrauch). Es existieren kaum Surveys, die zwischen diesen Konsummustern unterscheiden und diese genauer erfassten. 


\section{Charakteristik von Cannabis}

Die Hanfpflanze (Cannabis sativa L.) und ihre Produkte werden seit Jahrtausenden durch den Menschen zu religiösen, medizinischen und berauschenden Zwecken genutzt. Hanf gehört ebenso wie die Gattung Humulus (Hopfen) zu der Familie der Cannabaceae (Hanfgewächse) [6]. Ursprünglich stammt die Hanfpflanze aus Zentralasien, ist aber heute in fast allen warmen und gemäßigten Zonen der Erde verbreitet.

Merke

Cannabis gehört zu den ältesten Rauschmitteln und besitzt eine lange Tradition als Nutz- und Heilpflanze.

Ihre Rauschwirkung wurde in Europa schon im 19. Jahrhundert bekannt, der verbreitete Konsum begann in den 1970er-Jahren.

Im Cannabis wurden bisher etwa 500 chemische Substanzen gefunden, über 100 davon zählen zu der Gruppe der Cannabinoide [7]. Den Hauptwirkstoff stellt das THC dar. Seine chemische Struktur wurde bereits 1964 geklärt [8]. Daneben finden sich in der Pflanze weitere Cannabinoide, wie beispielsweise Cannabidiol (CBD) oder Cannabinol (CBN), sowie Nichtcannabinoide, wie z. B. Terpenoide, Flavonoide und Stickstoffverbindungen [9].

Pflanzliches Cannabis verfügt über verschiedene psychotrope Effekte: Stimmungssteigerung, Entspannung, Sedierung, veränderte Wahrnehmung akustischer und optischer Reize, Logorrhoe, Verminderung von Reaktionszeit und Konzentration, Gleichgültigkeit und Denkstörungen. Es können auch unangenehme Effekte auftreten: Gedankensprünge, Verwirrtheit, „Filmrisse“, Depressivität, Angst, Panik oder psychotische Symptome.

\section{Merke}

Die berauschende Wirkung der Pflanze geht insbesondere zurück auf den Hauptwirkstoff THC

( $\Delta 9$-Tetrahydrocannabinol).

THC ist das Agens mit der höchsten psychoaktiven Potenz, dessen Gebrauch zu einer akuten Intoxikation führen kann, die jedoch nicht tödlich ist. Im somatischen Bereich können durch Gebrauch von Cannabis auftreten: gesteigerter Appetit und Libido, Mundtrockenheit, Muskelentspannung, Schwindel, Übelkeit, Erbrechen, Hyperreflexie, Hyperstimulation mit Anstieg von Puls, Erweiterung der Blutgefäße, Mydriasis und konjunktivale Injektion. THC hat darüber hinaus schmerz- und übelkeitslindernde und muskelentspannende Wirkungen [10].

Der zweitwichtigste Hauptwirkstoff ist Cannabidiol (CBD). Ihm werden u. a. anxiolytische, antipsychotische, antiinflammatorische, antiemetische und neuroprotektive Effekte zugeschrieben [10]. CBD gilt in niedrigen Dosen als nicht intoxikierend, hat aber auch eine psychoaktive Wirkung, da es im Gehirn funktionelle Veränderungen erzeugen kann, die gegenläufig zu denen von THC sind [11].

\section{PRAXISTIPP}

Wie ein Mensch auf Cannabis reagiert, ist individuell sehr unterschiedlich und hängt von verschiedenen Faktoren wie Stimmungslage, Veranlagung, Konsumart, Konsumerfahrung, Situation sowie Menge, Intensität, Konsumdauer, Art sowie THC:CBD-Verhältnis der Cannabisprodukte ab.

\section{FALLBEISPIEL}

Leon (Schüler, 17 Jahre) berichtet, dass er vor einem halben Jahr zum ersten Mal Cannabis geraucht habe. Da einige seiner Klassenkameraden bereits gekifft hätten, sei er neugierig auf die Wirkung der Droge gewesen. Am Wochenende auf einer Party habe er dann zum ersten Mal mit Freunden einen Joint geraucht. Zuerst habe er sich euphorisch gefühlt. Alles sei absolut komisch gewesen, er habe über Kleinigkeiten herzhaft lachen können. Diese Euphorie habe dann aber schnell nachgelassen. Er habe nicht mehr klar denken können und das Gefühl gehabt, die Kontrolle über sich selbst zu verlieren. Dies habe ihm Angst gemacht. Ein Freund habe ihn dann nach Hause gebracht.

\section{Das endocannabinoide System}

Ende der 1980er-Jahre haben internationale Wissenschaftler das sogenannte Endocannabinoidsystem entdeckt. Wie bei der Erforschung des Opioidsystems in den 1970er-Jahren führte auch hier die Untersuchung von psychoaktiven Substanzen pflanzlichen Ursprungs zur Entdeckung dieses bis dahin unbekannten physiologischen Kontrollsystems.

\section{Cannabisrezeptoren}

Das Auffinden verschiedener Cannabinoidrezeptoren im zentralen und peripheren Nervensystem und körpereigener Cannabinoide (z. B. des Anandamids [N-arachidonoylethanolamide], revolutionierte den Forschungsbereich [12].

\section{Merke}

Der häufigste Cannabisrezeptor (Typ 1) ist vor allem im zentralen und weniger im peripheren Nervensystem verbreitet.

Eine besonders hohe Dichte des Cannabisrezeptors Typ 1 besteht im Gehirn, vor allem in Regionen, die mit der Körperbewegung, Lernen, Gedächtnis, Schmerz, Appetit, Übelkeit oder dem sogenannten Belohnungssystem assoziiert sind. 
Ein weiterer Cannabisrezeptor (Typ 2) ist im Gehirn weniger und in peripheren Organen und Organsystemen weit verbreitet. Er wurde vor allem in Organen des Immunsystems gefunden.

\section{Wirkmechanismen}

Die 2 Hauptbestandteile in Cannabis, THC und CBD, haben vermutlich gegensätzliche Effekte. THC aktiviert als partieller Agonist sowohl den Cannabisrezeptor Typ 1 als auch den Cannabisrezeptor Typ 2 [13]. Es beeinflusst die Aktivität von G-Proteinen und interagiert mit verschiedenen Neurotransmittersystemen, insbesondere Dopamin, Serotonin und GABA. Durch die CB1-Aktivierung kommt es zur Hemmung vorwiegend inhibitorischer Neurone (d.h. Enthemmung) und damit zu verstärkter dopaminerger Übertragung (u. a. im Nucleus accumbens). CBD hat nur eine geringe Affinität, an beide Endocannabinoidrezeptoren zu binden. Als Modulator reduziert es möglicherweise die Wirkung der Agonisten, eventuell wird aber auch die Zahl der Endocannabinoide erhöht [12]. CBD scheint aber über die CB2-antagonistische Wirkung antiinflammatorisch, anxiolytisch und antiemetisch zu wirken.

\section{Risiken von Cannabis zu Rauschzwecken}

In der wissenschaftlichen Literatur sind unterschiedlichste Effekte des Gebrauchs von Cannabis zu Rauschzwecken untersucht worden.

\section{THC:CBD-Verhältnis}

THC wird mit psychoseartigen Symptomen [14], [15], kognitiven Einschränkungen und gehirnstrukturellen Veränderungen bei langjährigen Cannabiskonsumenten in Verbindung gebracht [16], [17]. Hochpotentes THC in Cannabisprodukten wird bei gesunden Menschen als Auslöser für psychoseartige Symptome oder bei vulnerablen Menschen als Auslöser für Psychosen diskutiert [18], [19].

\section{Merke}

Studien aus dem Tier- und Humanbereich zeigten, dass CBD die adversen Effekte von THC verbessern kann [20].

Experimentelle Studien demonstrieren, dass die Gabe von oralem CBD vor einer intravenösen THC-Administration kognitive Einschränkungen und paranoide Effekte reduzierte [21]. Eine gleichzeitige THC-CBD-Infusion blockierte die durch THC-ausgelöste Ängstlichkeit und subjektive Veränderungen [22]. Eine experimentelle Studie von Solowij und Kollegen belegt, dass niedrige Dosierungen von CBD die Effekte von THC verstärken, während hohe Dosen von CBD die intoxikierenden Effekte von THC vermindern [11].

\section{THC-reiche Zuchtformen}

Durch spezifische Züchtungen in Gewächshäusern und durch professionelle Anbaumethoden (künstliche Beleuchtung, Spezialdüngung und Hydrosysteme) kann der Hauptwirkstoff THC in Cannabisprodukten gesteigert werden. Eine Übersichtsarbeit von Chandra und Kollegen zeigt, dass sich die durchschnittliche THC-Konzentration in Cannabisprodukten in den USA in den letzten 10 Jahren fast verdoppelt hat [9]: Sie ist von 8,9\% (2008) auf 17,1\% (2017) angestiegen. Auch das durchschnittliche Verhältnis von THC zu CBD hat sich sehr stark von $23 \mathrm{im} \mathrm{Jahr}$ 2008 auf 104 im Jahr 2017 erhöht. Für den US-amerikanischen Markt wurde ebenfalls eine markante Zunahme des durchschnittlichen THC-Gehalts (6,7-55,7\%) dokumentiert (2008-2017). Cannabismonitoring-Programme in den Niederlanden, Großbritannien, Frankreich, Italien und Australien belegen diese Entwicklung.

Auch in Deutschland ist im letzten Jahrzehnt der THCGehalt von Cannabisprodukten deutlich angestiegen. Dies zeigt der aktuelle Drogenbericht der Bundesregierung anhand von Daten des Bundeskriminalamtes [23]. Bei Haschisch (Cannabisharz) hat sich der THC-Gehalt zwischen 1996 und 2017 verdreifacht (von 4,9 auf $14,7 \%)$. Bei Marihuana hat sich der THC-Gehalt mehr als verdoppelt (1996: 4,8\%, 2017: 13,1\%). Experten befürchten, dass der hohe THC-Gehalt bei gleichzeitig niedrigem CBD-Gehalt in Cannabisprodukten das Risiko für gesundheitliche Schäden erhöht.

\begin{abstract}
Merke
Viele hochgezüchtete Cannabissorten enthalten heute nur noch sehr wenig oder kein CBD. Die Wirkung von THC kann dann nicht abgemildert werden, die gesundheitlichen Risiken steigen.
\end{abstract}

\section{Psychische, körperliche und soziale Folgen}

\section{Kognitive Funktionsdefizite}

Die aktuelle Forschungslage deutet darauf hin, dass ein langjähriger intensiver Cannabiskonsum die Hirnleistung und insbesondere das Gedächtnis verschlechtern kann [24]. Abhängig vom Konsumverhalten zeigen sich zum Teil erhebliche Beeinträchtigungen bei der Lern- und Erinnerungsleistung, aber auch negative Auswirkungen auf andere kognitive Fähigkeiten wie die Aufmerksamkeit, das Problemlösen und die Denkleistung. Auswirkungen auf die Intelligenz wurden aufgezeigt und kontrovers diskutiert [25], [26]. Menschen, die intensiv Cannabis konsumieren, brechen statistisch häufiger die Schule ab, besuchen seltener eine Universität und haben seltener akademische Abschlüsse als ihre Altersgenossen, die nicht Cannabis konsumieren [24]. Einbußen im Bildungserfolg zeigen sich vor allem, wenn Jugendliche bereits vor dem 15. Lebensjahr mit dem Cannabisgebrauch begonnen haben und über Jahre hinweg viel Cannabis konsumierten. 
Die wissenschaftliche Literatur hat Hinweise dafür, dass kognitive Funktionsdefizite möglicherweise nach längeren Abstinenzphasen umkehrbar sind [24]. Es sollte in künftigen Studien geklärt werden, ob und nach welcher Zeit der Abstinenz sich die Symptome wieder bessern. Ebenso ungeklärt ist, welche Rolle ein junges Einstiegsalter und geschlechtsspezifische Unterschiede dabei spielen [24], [27].

\section{Psychische Störungen}

In der Regel sind immer mehrere Risikofaktoren an der Ätiologie einer psychischen Störung beteiligt. Die Substanz Cannabis scheint - darauf deutet die wissenschaftliche Evidenz hin-jedoch die Wahrscheinlichkeit für vulnerable Personen zu erhöhen, an depressiven Störungen, Angststörungen und bipolaren Störungen zu erkranken [28]. Am deutlichsten ausgeprägt ist das erhöhte Krankheitsrisiko bei psychotischen Störungen [28], [29]: Bei gelegentlichem Konsum ist das Risiko um das 1,4- bis 2-Fache erhöht, bei intensivem Konsum steigt es um das 2- bis 3,4-Fache an. Cannabiskonsumenten erkranken in der Regel rund 2,7 Jahre früher erstmalig an der psychotischen Störung und haben einen ungünstigeren Krankheitsverlauf als Nichtkonsumenten [30]. Werden die Patienten abstinent, unterscheidet sich die Rückfallquote jedoch nicht mehr von Patienten, die nie Cannabis konsumiert haben.

Merke

Cannabis ist bei vulnerablen Personen ein Risikofaktor für spätere psychische Erkrankungen [28].

\section{Somatische Erkrankungen}

Neben den bereits erwähnten Risiken für die psychische Gesundheit birgt ein chronischer Cannabiskonsum auch eine größere Wahrscheinlichkeit für körperliche Erkrankungen, so z. B. Atemwegserkrankungen und Hodenkrebs (Nichtseminome, [31]). Das Risiko für Lungenkrebs oder Tumoren im Kopf-Hals-Bereich scheint dagegen nicht erhöht zu sein. $\mathrm{Zu}$ anderen Krebsarten ist die Datenlage zu dünn, um das Krankheitsrisiko beurteilen zu können. Bezüglich Herz- und Gefäßerkrankungen liegen keine ausreichenden Daten für die Auswirkungen eines chronischen Cannabiskonsums vor [31]. Cannabiskonsum während der Schwangerschaft kann Risiken für Mutter und Kind bergen [31].

\section{Cannabiskonsumstörungen}

Prävalenz

Etwa 9\% aller Cannabiskonsumenten entwickeln eine cannabisbezogene Störung [32]. Beginnt der Cannabiskonsum schon in der Adoleszenz, steigt die Rate auf $17 \%$ und auf 25-50\% bei täglichem Konsum. Europaweit wird die Anzahl der Menschen mit Cannabisabhängigkeit auf mindestens 3000000 Menschen geschätzt [33]. Für die deutsche erwachsene Allgemeinbevölkerung wird ein klinisch relevanter Cannabiskonsum bei 1,4\% der Männer und 1,0\% der Frauen geschätzt. Hochgerechnet auf die Bevölkerung im Alter von 18 und 64 Jahren waren damit im Jahr 2015 etwa 612000 Personen betroffen [4].

\section{Ätiologie}

Bei der Ätiologie von Cannabiskonsumstörungen handelt es sich, wie auch bei anderen Abhängigkeitserkrankungen, um ein multifaktorielles Geschehen [28]. Ein breites Spektrum an Personenfaktoren (z. B. Genetik, Persönlichkeit, Alter, Geschlecht), sozialen Faktoren (z. B. Peergroup), individuellen Faktoren (z.B. Stressoren) und Konsumgewohnheiten (z. B. Dosis, Frequenz, Potenz, KoKonsum mit Tabak) kann in Interaktion mit der individuellen Lerngeschichte (Modelllernen, operante und klassische Konditionierung) die Entwicklung von Cannabiskonsumstörungen bedingen. Das Vorliegen einer Major Depression oder psychotischen Störung erhöhen das Risiko einer Cannabisabhängigkeit, bei anderen psychischen Störungen sind die Ergebnisse wissenschaftlicher Studien heterogen [28].

Als mögliche kurzfristige Folgen der Cannabisgebrauchsstörung können sich beispielsweise zeigen: Probleme in der Schule bzw. am Arbeitsplatz oder in sozialen Beziehungen. Längerfristige negative Entwicklungen können sein: Isolation, Schulden oder sekundäre psychische Probleme wie Depressivität oder Ängstlichkeit.

Die jährliche Remissionsrate bei Cannabisabhängigkeit wird global auf $17,3 \%$ geschätzt [34]. Sie ist höher als die Remission bei Amphetamin- (16,4\%), Opiat- (9,2\%) und Kokainabhängigkeit (5,3\%).

\section{Diagnostik}

\section{FALLBEISPIEL}

Herr P. (35 Jahre) ist als Softwareentwickler seit 2 Jahren in einem Startup-Unternehmen tätig. Er habe es spannend gefunden, neue Ideen für Produkte zu entwickeln. Nicht selten arbeite er 60 Stunden pro Woche, in der Regel bis spät in die Nacht. Er habe schon seit langer Zeit Cannabis gebraucht, es helfe ihm zu entspannen. Im Unternehmen gebe es durch rasch wechselnde „Ups“ und „Downs“ Spannungen. Um den Frust zu vergessen, konsumiere er mehr Cannabis. Seit einiger Zeit gebe es Konflikte mit seiner Partnerin. Sie wünsche sich mehr gemeinsame Zeit und Aktivitäten. Es störe sie, dass er sich immer mehr zurückziehe. Morgens könne er schlecht aufstehen und abends ohne Joint nicht einschlafen. Er sei insgesamt unkonzentrierter geworden. Er wisse, dass er momentan zu viel kiffe. Es gelänge ihm aber nicht, etwas daran zu ändern. 
Die derzeit gültigen Klassifikationssysteme ICD-10 und DSM-5 definieren Kriterien für Störungen im Zusammenhang mit psychotropen Substanzen. In Deutschland werden Probleme im Zusammenhang mit dem Konsum von Alkohol, Tabak oder anderen psychotropen Substanzen (Cannabinoide, Sedativa oder Hypnotika, Kokain, Stimulanzien, Halluzinogene, flüchtige Lösungsmittel) nach der ICD-10 diagnostiziert. Kapitel F1 beschreibt verschiedene „psychische und Verhaltensstörungen durch psychotrope Substanzen“. Dies kann z. B. eine akute Intoxikation (d. h. ein akuter Rausch), ein schädlicher Gebrauch (F12.1), ein Abhängigkeitssyndrom (F12.2), ein Entzugssyndrom (F12.3), oder eine psychotische Störung (F12.5) sein ( $\triangleright$ Tab. 1$)$.
Klinisch immer wieder beobachtet wird das sogenannte amotivationale Syndrom. Es ist gekennzeichnet durch verminderte Motivation für Alltagsaktivitäten, Konzentrations- und Aufmerksamkeitsstörungen sowie affektive Verflachung. In der wissenschaftlichen Literatur ist das amotivationale Syndrom kontrovers diskutiert worden. Die Studien haben das Syndrom häufig unterschiedlich operationalisiert [35], was zu einer heterogenen Datenlage beigetragen hat. Es sollte künftig untersucht werden, wie ein solches Syndrom sinnvoll abgegrenzt und definiert werden kann.

All diese Störungen können unterschiedlichen Schweregrades und mit verschiedenen klinischen Erscheinungsbildern

- Tab. 1 Diagnostische Kriterien für eine Cannabisgebrauchsstörung (nach DSM-5) bzw. schädlichen und abhängigen Cannabisgebrauch (nach ICD-10).

\section{Cannabisgebrauchsstörung nach DSM-5}

Beim Auftreten von mindestens 2 Symptomen innerhalb eines 12-Monats-Zeitraums gilt die Substanzgebrauchsstörung als erfüllt.

1. Substanz wird häufig in größeren Mengen oder länger als geplant konsumiert (Kontrollverlust).

2. Anhaltender Wunsch oder erfolglose Versuche, den Substanzgebrauch zu verringern oder zu kontrollieren.

3. Hoher Zeitaufwand für Beschaffung und Konsum oder um sich von den Wirkungen des Konsums zu erholen.

4. Craving oder starkes Verlangen nach der Substanz.

5. Wiederholter Konsum, der zu einem Versagen bei der Erfüllung wichtiger Verpflichtungen in der Schule, bei der Arbeit oder zu Hause führt.

6. Fortgesetzter Substanzgebrauch trotz ständiger oder wiederholter sozialer oder zwischenmenschlicher Probleme.

7. Aufgabe oder Einschränkung wichtiger sozialer, beruflicher oder Freizeitaktivitäten aufgrund des Substanzkonsums.

8. Wiederholter Konsum in Situationen, in denen es aufgrund des Konsums zu einer körperlichen Gefährdung kommen kann.

9. Fortgesetzter Konsum trotz der Kenntnis von körperlichen oder psychischen Problemen, die durch den Substanzkonsum entstanden sind oder sich dadurch verschlechtert haben.

10. Toleranzentwicklung gekennzeichnet durch Dosissteigerung oder verminderte Wirkung unter derselben Dosis.

11. Entzugssymptome: a.) Ein für die Substanz spezifisches Entzugssyndrom, b.) Substanz (oder ähnliche Substanz) wird konsumiert, um Entzugssymptome zu lindern oder zu vermeiden. Die Schwere der Symptomatik wird folgend spezifiziert: Vorliegen von 2-3 Kriterien: mild; Vorliegen von 4-5 Kriterien: moderat; Vorliegen von mehr als 6 Kriterien: schwer.

\section{Substanzabhängigkeit nach ICD-10 (F1x.2)}

Drei oder mehr der folgenden Kriterien sollten zusammen mindestens einen Monat lang bestanden haben. Falls sie nur für eine kürzere Zeit gemeinsam aufgetreten sind, sollten sie innerhalb von 12 Monaten wiederholt bestanden haben.

1. Ein starkes Verlangen oder eine Art Zwang, die Substanz zu konsumieren.

2. Verminderte Kontrolle über den Substanzgebrauch, d. h. über Beginn, Beendigung oder die Menge des Konsums, deutlich daran, dass mehr von der Substanz konsumiert wird oder über einen längeren Zeitraum als geplant, und an erfolglosen Versuchen oder dem anhaltenden Wunsch, den Substanzkonsum zu verringern oder zu kontrollieren.

3. Ein körperliches Entzugssyndrom, wenn die Substanz reduziert oder abgesetzt wird, mit den für die Substanz typischen Entzugssymptomen oder auch nachweisbar durch den Gebrauch derselben oder einer sehr ähnlichen Substanz, um Entzugssymptome zu mildern oder zu vermeiden.

4. Toleranzentwicklung gegenüber den Substanzeffekten. Für eine Intoxikation oder um den gewünschten Effekt zu erreichen, müssen größere Mengen der Substanz konsumiert werden, oder es treten bei Konsum derselben Menge deutlich geringere Effekte auf

5. Einengung auf den Substanzgebrauch, deutlich an der Aufgabe oder Vernachlässigung anderer wichtiger Vergnügen oder Interessenbereiche wegen des Substanzgebrauchs; oder es wird viel Zeit darauf verwandt, die Substanz zu bekommen, zu konsumieren oder sich davon zu erholen.

6. Anhaltender Substanzgebrauch trotz eindeutig schädlicher Folgen, deutlich an dem fortgesetzten Gebrauch, obwohl der Betreffende sich über die Art und das Ausmaß des Schadens bewusst war oder hätte bewusst sein können.

\section{Schädlicher Gebrauch nach ICD-10 (F1x.1)}

A Deutlicher Nachweis, dass der Substanzgebrauch verantwortlich ist für die körperlichen oder psychischen Probleme, einschließlich der eingeschränkten Urteilsfähigkeit oder des gestörten Verhaltens, das evtl. zu Behinderung oder zu negativen Konsequenzen in den zwischenmenschlichen Beziehungen geführt hat.

B Die Art der Schädigung sollte klar bezeichnet werden können.

C Das Gebrauchsmuster besteht mindestens seit einem Monat oder trat wiederholt in den letzten 12 Monaten auf.

Auf die Störung treffen die Kriterien einer anderen psychischen oder Verhaltensstörung bedingt durch dieselbe Substanz, zum gleichen Zeitpunkt nicht zu (außer akute Intoxikation F1x.0). 
sein. Ihre Gemeinsamkeit besteht im Gebrauch einer oder mehrerer psychotroper Substanzen.

\section{Klassifikationssysteme}

Nach ICD-10 liegt ein schädlicher Substanzgebrauch (F1x.1) vor, wenn psychische oder körperliche Probleme mindestens einen Monat lang vorliegen oder im letzten Jahr wiederholt mehrfach auftraten und die Kriterien für eine Abhängigkeitsdiagnose noch nicht erfüllt sind.

Von einem Abhängigkeitssyndrom (F1x.2) spricht man, wenn mindestens 3 von 6 Kriterien während des letzten Jahres gleichzeitig vorhanden waren: starker Wunsch oder Zwang, psychotrope Substanzen zu konsumieren, verminderte Kontrollfähigkeit bezüglich des Beginns, der Beendigung oder der Menge des Konsums, körperliches Entzugssyndrom, Toleranz gegenüber der Substanz, fortschreitende Vernachlässigung anderer Vergnügungen oder Interessen, anhaltender Substanzkonsum trotz des Nachweises eindeutig schädlicher Folgen.

Während im DSM-IV auch noch zwischen den 2 Kategorien „Missbrauch“ und „Abhängigkeit“ unterschieden wurde, hat das DSM-5 diese Aufteilung zugunsten des dimensionalen Konzeptes einer „Substanzgebrauchsstörung“ aufgegeben. Diese wird durch 11 verschiedene Symptome beschrieben. Liegen 2 Merkmale innerhalb eines 12-Monats-Zeitraums vor, so gilt die Störung als erfüllt. Es gibt im DSM-5 nun auch die Möglichkeit, eine Schwere der Symptomatik zu spezifizieren. Beim Vorliegen von 2-3 Kriterien liegt eine „milde“, bei 4-5 Kriterien eine „moderate“, bei mehr als 6 Merkmalen eine „schwere“ Substanzgebrauchsstörung vor.

In der $>$ Tab. 1 finden sich die diagnostischen Kriterien der ICD-10 und des DSM-5 für schädlichen und abhängigen Substanzgebrauch bzw. für die Substanzgebrauchsstörung.
In beiden Klassifikationssystemen wird das Abhängigkeitssyndrom (ICD-10) bzw. die Substanzkonsumstörung (DSM-5) als ein sog. Symptomcluster definiert: Mehrere (mindestens 2 bzw. 3) unterschiedliche Symptome körperlicher, psychischer oder behavioraler Art müssen innerhalb eines definierten Zeitraums (12 Monate) aufgetreten sein, um die Diagnose stellen zu können.

\section{Klinische Symptomatik}

Klinisch ist das Syndrom heterogen. Es können ganz unterschiedliche Symptome im Vordergrund stehen. Ein zentrales Merkmal ist jedoch, dass eine Person trotz des Auftretens schwerwiegender Probleme nicht aufhört, eine oder mehrere psychotrope Substanzen zu konsumieren.

In beiden Klassifikationssystemen werden die Diagnosekriterien substanzübergreifend definiert.

\author{
- Merke \\ Menge und Häufigkeit des Substanzkonsums kommt \\ keinerlei diagnostische Bedeutung zu.
}

Während im ICD-10 [36] das Auftreten eines (unspezifischen) Entzugssyndroms bei abhängigen Cannabiskonsumenten bereits berücksichtigt wurde, waren Entzugssymptome bei Cannabisabhängigkeit in der Vorgängerversion des DSM-5 [37] formal nicht diagnostizierbar. Insgesamt wird das Entzugssymptom von Cannabinoiden als „leicht bis mittelgradig schwer“ beschrieben [1]. Es ähnelt dem von Tabak und anderen Substanzen und ist nicht lebensbedrohlich.

Mit der Neuauflage des amerikanischen Klassifikationssystems „Diagnostisches und Statistisches Manual Psychischer Störungen V“ (DSM-5) wurde erstmalig die Cannabiskonsumstörung als eigenständige Erkrankung mit cannabisspezifischem Entzugssyndrom aufgenommen ( $\triangleright$ Tab. 2) [37]. Es kann innerhalb von 48 Stunden nach dem Cannabiskonsumstopp auftreten. Mindestens

-Tab. 2 Cannabisentzugssymptome [38].

\begin{tabular}{|c|c|c|}
\hline Symptom & Dauer & Häufigkeit \\
\hline Ärger, Aggression, Irritation & wenige Tage bis 3 Wochen & sehr häufig \\
\hline Ängstlichkeit, Nervosität & wenige Tage bis 3 Wochen & sehr häufig \\
\hline Restlessness & wenige Tage bis 3 Wochen & sehr häufig \\
\hline Schlafschwierigkeit, bizarre Träume & wenige Tage bis 4 Wochen & sehr häufig \\
\hline Craving & wenige Tage & häufig \\
\hline Veränderung im Gewicht, weniger Appetit & meist in der ersten Woche & häufig \\
\hline Depressive Stimmung & unklar, meist 4 Wochen und länger & weniger häufig \\
\hline $\begin{array}{l}\text { körperliches Unwohlsein; Magenschmerzen, } \\
\text { Kälteschauer, Schwitzen, Unsicherheit / } \\
\text { Wackeligkeit }\end{array}$ & wenige Tage bis 3 Wochen & weniger häufig \\
\hline
\end{tabular}


2 psychische Beschwerden (z. B. Reizbarkeit, Unruhe, Ängstlichkeit, Depressivität, Aggressivität, Appetitverlust, Schlafprobleme) und mindestens 1 weiteres vegetatives Syndrom (z. B. Schwitzen, Zittern, Bauschmerzen, erhöhte Körpertemperatur, Kälteschauer, Kopfschmerzen) müssen für die Diagnosestellung vorliegen. Die Beschwerden sind in der ersten Woche am intensivsten und können bis zu einem Monat andauern.

Verglichen mit anderen Suchtstoffen liegt die Besonderheit von Cannabis in der langen Halbwertszeit von THC. Durch seine lipophilen Eigenschaften wird THC (ebenso wie weitere Cannabinoide) im Fettgewebe eingelagert und kann auch nach Beendigung des Cannabiskonsums noch mehrere Stunden bis Tage psychoaktiv an den Rezeptoren wirksam sein. Die Halbwertszeit für einen Gelegenheitskonsumenten beläuft sich dabei auf ca. 1,3 Tage, die eines regelmäßigen Konsumenten auf 5-13 Tage. Zudem kann die Ausübung physischer Aktivitäten (z. B. Radfahren) zur Lipolyse führen und dadurch den Plasmagehalt von THC bei ehemaligen Konsumenten erhöhen.

Dementsprechend verläuft der Entzug für Cannabis bei akutem Konsumstopp weniger abrupt als bei dem Konsum anderer psychotroper Substanzen, welche schneller aus dem Körper eliminiert werden und demzufolge nicht mehr an den jeweiligen Rezeptoren verfügbar sind.

PRAXISTIPP

Im Gegensatz zum Entzug von Alkohol oder Opiaten existieren bei der Entgiftung von Cannabis keine lebensbedrohlichen Risiken. In vielen Fällen kann sie problemlos im ambulanten Behandlungssetting durchgeführt werden [1].

\section{Behandlung}

\section{Behandlungsnachfrage in Europa}

In Europa ist die Behandlungsnachfrage aufgrund der Primärsubstanz Cannabis im letzten Jahrzehnt kontinuierlich angestiegen [3]. Im Jahr 2017 begaben sich 155000 Menschen in Europa eine Drogenbehandlung wegen ihres Cannabisgebrauchs. 83000 Menschen waren dabei zum ersten Mal in Behandlung. In 25 EU-Ländern mit verfügbaren Daten stieg die Gesamtzahl an Erstbehandlungen zwischen 2006 und 2017 um $76 \%$ an [3]. Verschiedenste Faktoren werden im Zusammenhang mit diesem Anstieg diskutiert: Eine Zunahme des Cannabisgebrauchs in der Bevölkerung, mehr intensive Konsumenten, eine höhere Verfügbarkeit von hochpotenten Cannabisprodukten, eine veränderte Wahrnehmung der Risiken, mehr Behandlungsangebote für Cannabisabhängige und mehr Zuweisung zu Behandlung (vor allem aus dem Bereich der Justiz) [39].
Etwa die Hälfte aller erstmals wegen Cannabis behandelten Patienten berichteten einen täglichen Konsum. Experten schätzen, dass in Europa nur 5-10\% der täglichen Konsumenten in entsprechende Behandlung kommen [33]. Ähnliche Angaben machen auch Stephens und Kollegen [40]: Von allen Menschen, die im Jahr 2003 die diagnostischen Kriterien eines schädlichen Cannabiskonsums oder einer Abhängigkeit erfüllten, waren nur 9,8\% in Behandlung.

Merke

Nur etwa jeder 10. Cannabisabhängige unterzieht sich einer Behandlung.

\section{Evidenzbasierte psychotherapeutische Behandlungsansätze}

Eine Expertise der Europäischen Drogenbeobachtungsstelle befasst sich mit evidenzbasierten Behandlungsmöglichkeiten für Kinder, Jugendliche und Erwachsene mit Cannabiskonsumstörungen [33]. In einer systematischen Literaturrecherche wurden 65 Publikationen ausgewertet (26 Reviews, 39 Studien).

\section{Kinder und Jugendliche}

25 Studien befassen sich speziell mit Interventionen für junge Cannabiskonsumenten. Alle Interventionen richten sich an junge Menschen, die sich in einem frühen Stadium des problematischen oder abhängigen Cannabiskonsums befinden. Sie greifen das individuelle Risikoverhalten, den Drogenkonsum und damit verbundene psychische, körperliche und soziale Probleme eines Jugendlichen auf. Als wirksam haben sich motivationale Interventionen [41], [42], kognitiv-behaviorale Therapie (KBT) und Kontingenzmanagement (KM) erwiesen. Meist werden diese Ansätze kombiniert, die Programme haben in der Regel bis zu 10 Sitzungen. Ebenfalls erfolgversprechend sind familientherapeutische Behandlungsansätze (z. B. die multidimensionale Familientherapie), in denen die Jugendlichen und ihr familiäres, schulisches und soziales Umfeld in die Behandlung einbezogen werden. Die familientherapeutische Behandlung ist in der Regel umfangreich in Stundenzahl und Dauer, variiert jedoch auch je nach therapeutischer Strategie und Zielsetzung des Behandlungsprogramms.

\section{Ältere Jugendliche und Erwachsene}

Psychotherapeutische Interventionen, die auf den therapeutischen Strategien der Motivationsförderung, kognitiv-behavioralen Therapie und/oder Kontingenzmanagement beruhen, sind in der Zielgruppe der über 16-Jährigen am effektivsten. In der Europäischen Cannabisexpertise wurden 19 Studien ausgewertet (10 Reviews/Metaanalysen, 8 randomisiert-kontrollierte Studien, eine quasi-experimentelle Studie). Sie belegen, dass für Erwachsene mit einer primären Cannabisgebrauchsstörung Kombinationen aus Motivationsförderung (MET), kognitiv-behavioraler Therapie 
(CBT) und Kontingenzmanagement die höchste Effektivität aufweisen. Auch andere Übersichtsarbeiten und Expertisen belegen diese Aussage [43], [44], [45].

\section{Cannabis und komorbide psychische Erkrankungen}

Evidenzbasierte Behandlungsansätze für Menschen mit komorbiden Psychosen oder Schizophrenie, affektiven Erkrankungen, substanzbezogenen Störungen, Aufmerksamkeitsdefizit- und Hyperaktivitäts- oder Persönlichkeitsstörungen fehlen bislang nahezu vollständig. Sie benötigen vermutlich eine längere und intensivere Behandlung, die beide Erkrankungen berücksichtigt und durch Pharmakotherapie ergänzt wird.

\section{FALLBEISPIEL}

Herr M. (21 Jahre alt) kommt in Begleitung seiner besorgten Eltern in die Klinik. Er berichtet über innere Leere, reduzierten Antrieb, fehlende Energie. Seit ein paar Wochen fühle er sich ständig beobachtet, man verfolge ihn. „Irgendetwas braut sich zusammen“, meint er. Um seine Anspannung und Angst zu reduzieren, konsumiere er Cannabis. Die Eltern berichten, dass der Patient vor 2 Jahren eine erste psychotische Episode gehabt habe. Damals habe er 2 verschiedene antipsychotische Medikamente erhalten, diese aber jeweils wegen Nebenwirkungen (motorische Unruhe, Appetitsteigerung) wieder eigenständig abgesetzt. Eine angebahnte Psychotherapie konnte er damals aufgrund des Antriebsmangels nicht wahrnehmen.

\section{Effektivität der Behandlungsansätze}

Die bisher entwickelten Behandlungsprogramme sind wirksam, die Effekte jedoch eher moderat. In einem Cochrane Review von Gates und Kollegen berichteten 8 von 23 Studien Abstinenz (Punkt-Prävalenzen) [44]. Durchschnittlich $37 \%$ der Teilnehmer an den Interventionsgruppen waren zu Therapieende abstinent. Dieser Anteil reduzierte sich auf 24\% nach 3-4 Monaten und lag bei $23 \%$ bei längeren Follow-up-Zeiträumen. Die Abstinenzrate lag in den Kontrollgruppen bei der letzten Nachuntersuchung durchschnittlich bei $12 \%$. In den ausgewerteten Studien wurden zusätzlich auch weitere, sekundäre Therapieerfolge berichtet, z. B. Verbesserungen von Häufigkeit und Intensität des Cannabiskonsums. Daraus ergibt sich ein großer Bedarf an der Entwicklung evidenzbasierter Standards für die Behandlung der Cannabisabhängigkeit [45].

\section{Merke}

Die bisher durchgeführten Psychotherapiestudien zeigen moderate Abstinenzraten und hohe Rückfallraten bei Cannabiskonsumstörungen.

\section{Pharmakotherapie}

Bisher gibt es keine Arzneimittel, die für die Behandlung von Cannabiskonsumstörungen zugelassen sind. In einem Cochrane-Review analysierten Nielsen und Kollegen 21 randomisierte, kontrollierte Studien (1755 Teilnehmer) [46]. Alle untersuchten Substanzen hatten im Vergleich zum Placebo keine Effekte auf die Abstinenzraten: Cannabinoidrezeptor-1-Agonisten (THC-Präparationen; Nabiximols, Dronabiol), Antidepressiva (Selective Serotonin Reuptake Inhibitors (SSRI), Antidepressiva mit anderen Wirkmechanismen), Antikonvulsiva and Mood Stabiliser, Buspiron und N-Acetylcystein. Bei Gabapentin (Antikonvulsivum), Oxytocin (Neuropeptide) und Atomoxetin wurde die Datenlage als zu gering eingeschätzt, um Wirksamkeitsnachweise zu evaluieren. Es kam nicht häufiger zu Nebenwirkungen als bei Placebogabe. In 3 Studien (141 Teilnehmer) führte die Gabe von Antikonvulsiva und Mood Stabilisern zu frühzeitigem Behandlungsabbruch (RR 0,66, 95 \%-KI 0,47-0,92).

\section{Merke}

Es existieren keine Arzneimittel zur Behandlung des Cravings oder zur Verbesserung der Rückfallraten bei Cannabiskonsumstörungen.

\section{Cannabiskonsumstörungen im deutschen Gesundheitssystem}

In Deutschland ist Cannabis nach Alkohol der häufigste Anlass für eine Suchtbehandlung. Im Jahr 2016 wurden in der ambulanten Behandlung 48,9\% aller Fälle wegen Alkohol behandelt, 17,8\% wegen Cannabis und 13,7\% wegen Opiaten. In der stationären Behandlung war der Anteil von Alkoholbehandlungen noch größer (68,8\%). Wegen Cannabis waren 9,2\% in Behandlung, wegen Opiaten $5,2 \%$ [23].

In Deutschland - wie auch in anderen europäischen Ländern - werden cannabisbezogene Störungen in der Regel ambulant behandelt [1]. Dies erfolgt in Suchtberatungsstellen, Suchtambulanzen oder Schwerpunktpraxen. Auch der meist klinisch unkomplizierte Entzug kann ambulant erfolgen.

\section{PRAXISTIPP}

Kompliziertere Intoxikationsverläufe können durch Panikattacken, psychotische oder delirante Symptome gekennzeichnet sein. In diesen Fällen sind Gespräche mit dem Patienten und gegebenenfalls eine zeitlich limitierte Gabe von Antipsychotika (vorzugsweise Atypika) und / oder Sedativa hilfreich.

Eine qualifizierte stationäre Behandlung findet dann statt, wenn ein Betroffener einen komplizierten 
Intoxikationsverlauf, ein schweres Entzugssyndrom oder schwere psychische, somatische und soziale Folgestörungen, komorbide Störungen oder eine hohe Rückfallgefährdung mitbringt [47].

Die Therapie besteht dabei aus:

- Akutbehandlung und

- Postakutbehandlung.

In der Akuttherapie (Dauer: 2-4 Wochen; bei Jugendlichen 4-12 Wochen) kann eine körperliche Entgiftung, Diagnostik, Behandlung von Entzugssymptomen sowie Abklärung und gegebenenfalls Behandlung komorbider Störungen erfolgen. Neben intensiven stützenden Gesprächen und Tagesstrukturierung wird der Patient dazu motiviert, eine weiterführende Behandlung aufzunehmen, wenn bei beeinträchtigtem psychosozialem Funktionsniveau (das heißt bei Schwierigkeiten in der Organisation des Alltags und der Tagesstrukturierung) der Behandlungswille noch fehlt. In stationären Einrichtungen kommen verschiedene Therapieelemente zum Einsatz: Einzel- und Gruppentherapie, Rückfallmanagement, soziales Kompetenztraining, Entspannungsverfahren, Arbeits- und Beschäftigungstherapie, Physio- und Sporttherapie, soziale Hilfen, freizeitpädagogische Aktivitäten, bei Jugendlichen auch Unterrichtprogramme (Schule, Berufsorientierung, Suchtkunde).

Hinsichtlich der Behandlungsdauer haben sich 2 Varianten durchgesetzt:

- Kurztherapien mit einer Behandlungsdauer von 3 bis 6 Monaten und

- Langzeittherapien mit 12- bis 18-monatiger Behandlungsdauer.

Bei jüngeren Patienten mit erheblichen Entwicklungsdefiziten ist eine Langzeitbehandlung üblich [48].

Merke

Im Gegensatz zu alkoholbezogenen Störungen oder Opiatabhängigkeit sind in Deutschland bislang kaum Selbsthilfegruppen für Menschen mit cannabisbezogenen Störungen vorhanden.

\section{Evidenzbasierte Programme aus Deutschland}

Der erste in Deutschland entwickelte und mittlerweile auch in zahlreichen anderen europäischen Ländern angebotene Ansatz zur Behandlung von Cannabiskonsumstörungen ist „FreD - Frühintervention bei erstauffälligen Drogenkonsumenten“ [49]. Ziel dieses Programms ist es, junge Drogenkonsumenten zu einem möglichst frühen Zeitpunkt über Risiken zu informieren und zu einer Reflexion bzw. Veränderung des Substanzkonsums zu motivieren. Die Intervention besteht aus einem
Einzelgespräch (In-Take-Gespräch) und einem 8-stündigen Kursangebot (4-mal 2 Stunden bzw. 2-mal 4 Stunden).

Das Gruppentrainingsprogramm „CAN Stop“ wurde für Jugendliche und junge Erwachsene mit problematischem Cannabiskonsum im Alter zwischen 14 und 21 Jahren entwickelt, umgesetzt und evaluiert. Es kann von geschulten Laienhelfern in unterschiedlichen Settings (z. B. im Jugendstrafvollzug) eingesetzt werden [50].

Zur Behandlung von Jugendlichen mit problematischem Cannabiskonsum und multiplem Problemverhalten hat sich die Multidimensionale Familientherapie als wirksam gezeigt [51], [52]. Bei diesem Ansatz werden neben dem Jugendlichen auch die Eltern, die Familie und das soziale Umfeld in die Behandlung einbezogen. Es erfolgt eine Kombination von Einzelsitzungen (Jugendliche, Eltern), Familiensitzungen und Sitzungen mit relevanten außerfamiliären Bezugspersonen (z. B. Lehrer, Erzieher, Bewährungshelfer) in unterschiedlichen Settings (z. B. in der Einrichtung, zu Hause oder in der Schule). Es gibt keine spezifische Zeitbegrenzung für die Behandlung. Häufige telefonische Kontakte sind ein wichtiger Bestandteil der Intervention.

Für Cannabiskonsumenten im Alter zwischen 15 und 30 Jahren, die ihren Konsum einstellen, zumindest aber reduzieren wollen, wurde das internetbasierte Cannabisausstiegsprogramm „Quit the Shit“ entwickelt [53]. Nach dem Aufnahmegespräch wird das Online-Tagebuch von „Quit the shit" freigeschaltet. In diesem passwortgeschützten, persönlichen Bereich können die Teilnehmer über einen Zeitraum von 50 Tagen alle relevanten Aspekte ihres Cannabiskonsums festhalten. RegelmäBig-d. h. einmal pro Woche-erhalten alle Teilnehmer hierauf qualifizierte Feedbacks, in denen neben dem Konsum (aktuell, im Verlauf) auch die psychosoziale Situation der teilnehmenden Person oder der Beratungsprozess thematisiert werden.

Das Beratungsprogramm „Realize it“ richtet sich an 15bis 30-jährige Klientinnen und Klienten [54]. Das Ziel ist eine signifikante Reduktion des Cannabiskonsums (konsumfreie Tage, Konsumpausen oder die Cannabisabstinenz). Das „Realize it“-Programm wird vorwiegend in ambulanten Suchthilfeeinrichtungen in der Beratung eingesetzt. Seit 2016 ist eine Kombination der persönlichen Vor-Ort-Beratung mit einer webbasierten Variante des „Realize it“-Programms verfügbar. Die Teilnehmenden können über ihr Smartphone Zugang zu ihrem persönlichen Begleitbuch haben und mit ihren Beraterinnen bzw. Beratern in Kontakt treten.

Das CANDIS-Programm ist ein psychotherapeutischer Ansatz, ältere Jugendliche ( $\geq 16$ Jahre) und Erwachsenen mit cannabisbezogenen Störungen [55], [56], [57], [58]. 
Das Behandlungsprogramm besteht aus 10 Sitzungen und hat Abstinenz bzw. Konsumreduktion als Ziel. Es erfolgt Psychoedukation zum Thema Cannabis, Veränderungs- und Therapiemotivation werden aufgebaut, das Konsumverhalten der Teilnehmer wird analysiert. Es werden Fertigkeiten zur Veränderung des Cannabiskonsums eingeübt (z. B. Auslöserkontrolle, Aufbau von Verhaltensalternativen, Umgang mit Entzugsbeschwerden). Um den Therapieerfolg zu sichern, widmet sich das Programm den Themen Craving, Rückfallprophylaxe, Training sozialer Kompetenzen und Komorbidität mit anderen psychischen Störungen.

\section{Merke}

Das CANDIS-Manual wurde in mehrere Sprachen übersetzt und auch in anderen Ländern implementiert (z. B. in Polen, Spanien, Österreich und Italien).

\section{Schlussfolgerung}

In den letzten 3 Jahrzehnten hat die Forschung zu einem verbesserten Verständnis von Cannabis, den Cannabinoiden und dem körpereigenen Cannabissystem beigetragen. Die Studien zeigen, dass die Wirkungsweise von Cannabis individuell unterschiedlich sein kann. Empirisch gut gesichert ist, dass der biografisch frühe, intensive und langjährige Cannabisgebrauch das Risiko für unterschiedliche Störungen erhöht (z. B. kognitive Störungen, Einbußen im Bildungserfolg, Psychosen, Depressionen, bipolare Störungen, Angsterkrankungen sowie psychische und körperliche Abhängigkeit).

In der letzten Dekade zeigte sich parallel zum Anstieg des THC-Gehalts in Cannabisprodukten auch eine deutliche Zunahme in der Nachfrage nach Suchtberatung und -therapie wegen cannabisassoziierter Probleme in Europa. Experten schätzen, dass trotz dieser Entwicklung noch immer eine erhebliche Behandlungslücke für diese Klientel existiert. Die Erfolgsraten der bisher existierenden psychotherapeutischen Behandlungsansätze sind eher moderat, die Rückfallraten hoch. Effektive pharmakotherapeutische Optionen fehlen. Aus diesen Gründen kommt der Prävention von riskantem, schädlichem und abhängigem Cannabisgebrauch künftig eine tragende gesundheitspolitische Rolle zu.

\section{KERNAUSSAGEN}

- Nahezu 25 Mio. Bürger und Bürgerinnen in der EU nutzen Cannabis zu Rauschzwecken, ca. 3 Mio. von ihnen mit klinisch relevantem Konsum.

- Das durchschnittliche Verhältnis von THC zu CBD in Cannabisprodukten hat sich im letzten Jahrzehnt sehr stark erhöht. Dadurch steigen möglicherweise die gesundheitlichen Risiken von Cannabis, insbesondere bei vulnerablen Personen.
- Regelmäßiger und in der Adoleszenz beginnender Cannabiskonsum ist mit einem erhöhten Risiko für psychische Erkrankungen wie Depressionen und Psychosen verbunden, kann aber auch kognitive Fähigkeiten, den Bildungserfolg, die Fahrtüchtigkeit signifikant einschränken.

- Nach Alkohol ist der Konsum von Cannabis der häufigste Anlass für einen Kontakt mit dem Suchthilfesystem in Deutschland.

- Ein cannabisspezifisches Entzugssyndrom kann innerhalb von 48 Stunden nach dem Cannabiskonsumstopp auftreten. Es ist i. R. gut behandelbar und klinisch unkompliziert.

- Wirksame Therapieansätze zur Behandlung von Cannabiskonsumstörungen sind die motivationale Gesprächsführung in Kombination mit kognitiver Verhaltenstherapie.

- Für Kinder und Jugendliche mit pathologischem Cannabiskonsum wurden auch familientherapeutische Behandlungsansätze entwickelt.

- Die Behandlungserfolge sind eher moderat: Etwa jeder 4. Therapieteilnehmer ist nach 3-4 Monaten noch abstinent.

- Bisher existieren keine Arzneimittel, die für die Behandlung von Cannabiskonsumstörungen zugelassenen sind.

- Behandlungsansätze für Menschen mit Cannabiskonsumstörungen und komorbiden Erkrankungen (z. B. Psychosen, affektiven Erkrankungen, Aufmerksamkeitsdefizit- und Hyperaktivitäts- oder Persönlichkeitsstörungen) fehlen.

\section{Interessenkonflikt}

Eva Hoch erhielt Forschungsgelder vom BMG und der EMCDDA für Studien und Expertisen zum Thema Cannabis. Honorare für Vorträge von Einrichtungen und Organen des Gesundheitssystems, Desitin und Lundbeck. Ulrich W. Preuss gibt an, dass kein Interessenkonflikt besteht.

\section{Autorinnen / Autoren}

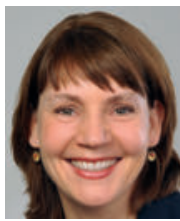

\section{Eva Hoch}

Priv. Doz. Dr. rer. nat. Sie ist approbierte Psychologische Psychotherapeutin und Leitende Psychologin an der Klinik für Psychiatrie und Psychotherapie, Klinikum der Universität München, Ludwig-Maximilians-Universität, München. Zu den Schwerpunkten ihrer klinischen und wissenschaftlichen Tätigkeit zählen die Erforschung, Diagnostik und Behandlung von Abhängigkeitserkrankungen, Affektiven Störungen, Psychosen und Persönlichkeitsstörungen. Besonderes Interesse: Erforschung der Wirkungsweise von Cannabinoiden auf die menschliche Gesundheit. 


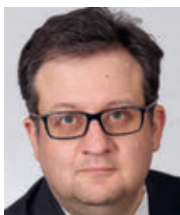

\section{Ulrich W. Preuss}

Prof. Dr. med. ist Facharzt für Psychiatrie und Psychotherapie und ärztlicher Direktor der Vitos Klinik für Psychiatrie und Psychotherapie, Herborn. Zu den Schwerpunkten seiner klinischen und wissenschaftlichen Tätigkeit zählen die Erforschung, Diagnostik und Behandlung von stoffgebundenen Abhängigkeitserkrankungen, Demenzen, Persönlichkeitsstörungen und schizophrenen Psychosen.

Korrespondenzadresse

Priv.-Doz. Dr. rer. nat. Eva Hoch

Klinik und Poliklinik für Psychiatrie und Psychotherapie Klinikum der Universität München Campus Innenstadt Nußbaumstraße 7

80336 München

Email: Eva.Hoch@med.uni-muenchen.de

www.klinikum.uni-muenchen.de

\section{Wissenschaftlich verantwortlich gemäß} Zertifizierungsbestimmungen

Wissenschaftlich verantwortlich gemäß

Zertifizierungsbestimmungen für diesen Beitrag ist PD Dr. Eva Hoch, München.

\section{Zitierweise für diesen Artikel}

PSYCH up2date 2019; 13 (5): 395-409

Literatur

[1] Hoch E, Petersen K, Thomasius R. Cannabis. In: Batra A, BilkeHentsch O, Hrsg. Praxisbuch Sucht: Therapie der Suchterkrankungen im Jugend- und Erwachsenenalter. Stuttgart: Thieme; 2016: 144-151

[2] United Nations Office on Drugs and Crime. World Drug Report. United Nations Office on Drugs and Crime, Vienna, Austria; 2018

[3] Europäische Beobachtungsstelle für Drogen und Drogenpolitik (EMCDDA). Europäischer Drogenbericht. Trends und Entwicklungen. Portugal: Lissabon; 2019

[4] Gomes de Matos E, Atzendorf J, Kraus L et al. Substanzkonsum in der Allgemeinbevölkerung in Deutschland. Ergebnisse des Epidemiologischen Suchtsurveys 2015. Sucht 2016; 62: 271-283

[5] Orth B. Die Drogenaffinität Jugendlicher in der Bundesrepublik Deutschland 2015. Rauchen, Alkoholkonsum und Konsum illegaler Drogen: aktuelle Verbreitung und Trends. BZgA-Forschungsbericht. Köln: Bundeszentrale für gesundheitliche Aufklärung; 2016

[6] Schneider M, Hoch E. Cannabis: Botanische, kulturelle und historische Aspekte. In: Hoch E, Friemel CM, Schneider M, Hrsg. Cannabis: Potenzial und Risiko. Eine wissenschaftliche Bestandsaufnahme. Heidelberg: Springer Nature; 2019: 2-3

[7] ElSohly MA, Mehmedic Z, Foster $S$ et al. Changes in cannabis potency over the last 2 decades (1995-2014): Analysis of current data in the United States. Biol Psychiatry 2016; 79: 613-619

[8] Gaoni Y, Mechoulam M. Isolation, structure, and partial synthesis of an active constituent of Hashish. J Am Chem Soc 1964; 86: 1646-1647

[9] Chandra S, Radwan MM, Majumdar CG et al. New trends in cannabis potency in USA and Europe during the last decade (2008-2017). Eur Arch Psychiatry Clin Neurosci 2019; 269: 5. doi:10.1007/s00406-019-00983-5

[10] Häuser W. Cannabis als Medizin. In Hoch E, Friemel CM, Schneider M, Hrsg. Cannabis: Potenzial und Risiko. Eine wissenschaftliche Bestandsaufnahme. Heidelberg: Springer Nature; 2019: 26-31

[11] Solowij N, Broyd S, Greenwood L et al. A randomised controlled trial of vaporised $\Delta 9$-tetrahydrocannabinol and cannabidiol alone and in combination in frequent and infrequent cannabis users: acute intoxication effects. Eur Arch Psychiatry Clin Neurosci 2019; 269: 17. doi:10.1007/s00406-019-00978-2

[12] Schneider M, Lutz B. Wirkweise von Cannabis: Aufbau und Funktion des Endocannabinoidsystems. In: Hoch E, Friemel CM, Schneider M, Hrsg. Cannabis: Potenzial und Risiko. Eine wissenschaftliche Bestandsaufnahme. Heidelberg: Springer Nature; 2019: 3-17

[13] Pertwee RG. The diverse CB1 and CB2 receptor pharmacology of three plant cannabinoids: delta9-tetrahydrocannabinol, cannabidiol and delta9-tetrahydrocannabivarin. $\mathrm{Br} J$ Pharmacol 2008; 153: 199-215

[14] D'Souza DC, Perry E, MacDougall L et al. The psychotomimetic effects of intravenous delta-9-tetrahydrocannabinol in healthy individuals: implications for psychosis. Neuropsychopharmacology 2004; 29: 1558-1572

[15] Morrison PD, Zois V, McKeown DA et al. The acute effects of synthetic intravenous $\Delta 9$-tetrahydrocannabinol on psychosis, mood and cognitive functioning. Psychol Med 2009; 39: $1607-1616$

[16] Broyd S], van Hell HH, Beale C et al. Acute and chronic effects of cannabinoids on human cognition: a systematic review. Biol Psychiatry 2016; 79: 557-567

[17] Lorenzetti V, Solowij N, Yücel M. The role of cannabinoids in neuroanatomic alterations in cannabis users. Biol Psychiatry 2016; 79: e17-e31

[18] Di Forti M, Marconi A, Carra E et al. Proportion of patients in south London with first-episode psychosis attributable to use of high potency cannabis: a case-control study. Lancet Psychiatry $2015 ; 2: 233-238$

[19] Yücel M, Lorenzetti V, Suo C et al. Hippocampal harms, protection and recovery following regular cannabis use. Transl Psychiatry 2016; 6: e710

[20] Osborne AL, Solowij N, Weston-Green K. A systematic review of the effect of cannabidiol on cognitive function: relevance to schizophrenia. Neurosci Biobehav Rev 2017; 72: 310-324

[21] Englund A, Morrison PD, Nottage J et al. Cannabidiol inhibits THC-elicited paranoid symptoms and hippocampal-dependent memory impairment. J Psychopharmacol 2013; 27: 19-27

[22] Zuardi AW, Shirakawa I, Finkelfarb E et al. Action of cannabidio on the anxiety and other effects produced by delta 9-THC in normal subjects. Psychopharmacology (Berl) 1982; 76: 245-250

[23] Bundesministerium für Gesundheit (BMG). Drogen- und Suchtbericht. Im Internet: https: / /www.drogenbeauftragte. de/fileadmin/dateien-dba/Drogenbeauftragte/Drogen_und_ Suchtbericht/pdf/DSB-2018.pdf; Stand: 17.04.2019

[24] Friemel CM, Schneider M, Lutz B, Hermann D, Hasan A, Kambeitz J, Hoch E. Kognition. In: Hoch E, Friemel CM, Schneider 
M, Hrsg. Cannabis: Potenzial und Risiko. Eine wissenschaftliche Bestandsaufnahme. Heidelberg: Springer Nature; 2019: 66-95

[25] Meier MH, Caspi A, Ambler A et al. Persistent cannabis users show neuropsychological decline from childhood to midlife. Proc Natl Acad Sci U S A 2012; 109: E2657-E2664

[26] Rogeberg O. Correlations between cannabis use and IQ change in the Dunedin cohort are consistent with confounding from socioeconomic status. Proc Natl Acad Sci U S A 2013; 110: 4251-4254

[27] Gorey C, Kuhns L, Smaragdi E et al. Age-related differences in the impact of cannabis use on the brain and cognition: a systematic review. Eur Arch Psychiatry Clin Neurosci 2019; 269: 37-58

[28] Hoch E, von Keller R, Schmieder S, Friemel CM, Hermann D, Bonnet $U$, Preuss $U$, Schneider M. Affektive Störungen und Angststörungen. In: Hoch E, Friemel CM, Schneider M, Hrsg. Cannabis: Potenzial und Risiko. Eine wissenschaftliche Bestandsaufnahme. Heidelberg: Springer Nature; 2019: 213-232

[29] Scott JC, Slomiak ST, Jones JD et al. Association of cannabis with cognitive functioning in adolescents and young adults: A systematic review and meta-analysis. JAMA Psychiatry 2018; 75: 585-595

[30] Large M, Sharma S, Compton MT et al. Cannabis use and earlier onset of psychosis: a systematic meta-analysis. Arch Gen Psychiatry 2011; 68: 555-561

[31] Schneider M, Friemel C, Schäfer M, Storr M, Hoch E. Somatische Folgen. In: Hoch E, Friemel CM, Schneider M, Hrsg. Cannabis: Potenzial und Risiko. Eine wissenschaftliche Bestandsaufnahme. Heidelberg: Springer Nature; 2019: 95129

[32] Volkow ND, Baler RD, Compton WM et al. Adverse health effect of marijuana use. N Engl J Med 2014; 370: 2219-2227

[33] European Monitoring Centre for Drugs and Drug Addiction (EMCDDA). Treatment of cannabis-related Disorders in Europe. Luxembourg: Publications Office of the European Union; EMCDDA Insights No 17; 2015

[34] Calabria B, Degenhardt L, Briegleb C et al. Systematic reviews of prospective studies investigating "remission" from amphetamine, cannabis, cocaine and opioid dependence. Addict Behav 2010; 35: 741-749

[35] Lac A, Luk JW. Testing the amotivational syndrome: marijuana use longitudinally predicts lower self-efficacy even after controlling for demographics, personality, and alcohol and cigarette use. Prev Sci 2018; 19: 117-126

[36] Dilling H, Mombour W, Schmidt M, Hrsg. ICD-10. Kapitel V (F) Klinisch-diagnostische Leitlinien. 9. Aufl. Bern: Huber; 2013

[37] American Psychiatric Association. Diagnostic and statistical manual of mental disorders. 5th ed. Washington, DC: American Psychiatric Association; 2013

[38] Copeland C, Frewen A, Elkin K. Management of Cannabis Use Disorder and related Issues: a Clinician's Guide. University of New South Wales, Sydney: National Cannabis Prevention and Information Centre; 2009

[39] Montanari L, Guarita B, Mounteney J et al. Cannabis Use among People Entering Drug Treatment in Europe: A Growing Phenomenon? Eur Addict Res 2017; 23: 113-121

[40] Stephens RS, Roffman R, Fearer $S$ et al. The marijuana checkup: Promoting change in ambivalent marijuana users. Addiction 2007; 102: 947-957
[41] Miller W. Motivational Interviewing with Problem Drinkers. Behav Cogn Psychother 1983; 11: 147-172. doi:10.1017| S0141347300006583

[42] Miller WR, Rollnick S. Motivational Interviewing: Preparing People for Change. New York: Guilford Press; 2002

[43] Davis ML, Powers MB, Handelsman P et al. Behavioral therapies for treatment-seeking cannabis users: A meta-analysis of randomized controlled trials. Eval Health Prof 2015; 38: 94114

[44] Gates PJ, Sabioni P, Copeland J et al. Psychosocial interventions for cannabis use disorder. Cochrane Database Syst Rev 2016; (5): CD005336. doi:10.1002/14651858.CD005336.pub4

[45] Walther L, Gantner A, Heinz A et al. Evidence-based treatment options in cannabis dependency. Dtsch Arztebl Int 2016; 113: 653-659

[46] Nielsen S, Gowing L, Sabioni P et al. Pharmacotherapies for cannabis dependence. Cochrane Database Syst Rev 2019; (1): CD008940. doi:10.1002/14651858.CD008940.pub3

[47] Hoch E, Bonnet U, Thomasius R et al. Risiken bei nichtmedizinischem Gebrauch von Cannabis. Dtsch Arztebl Int 2015; 112: $271-278$

[48] Thomasius R, Stolle M. Diagnostik und Behandlung psychischer Störungen durch Cannabis. Psychiatrie und Psychotherapie up2date 2008; 2: 225-241

[49] Görgen W, Hartmann R, Oliva H. Frühintervention bei erstauffälligen Drogenkonsumenten-FreD. Abschlussbericht der wissenschaftlichen Begleitung. Forschungsbericht 299, Sozialforschung. Bonn: Bundesministerium für Gesundheit und Soziale Sicherung; 2003

[50] Baldus C, Kindermann SS, Stappenbeck ], Reis O, Thomasius R. CAN Stop: Psychoedukation und Rückfallprävention für junge Menschen mit problematischem Cannabiskonsum Entwickung und Evaluation eines Gruppenbehandlungsprogramms: Abschlussbericht an das Bundesministerium für Gesundheit (BMG). Hamburg: DZSKJ; 2011

[51] Liddle HA. Multidimensional Family Therapy for adolescent Cannabis Users. Cannabis Youth Treatment (CYT) Series, Volume 5. Rockville, MD: Center for Substance Abuse Treatment, Substance Abuse and Mental Health Services Administration; 2002

[52] Tossmann P, Jonas B, Rigter H. Multidimensionale Familientherapie (MDFT) bei cannabisbezogenen Störungen. Sucht 2012; 58: 157-166

[53] Tossmann HP, Jonas B, Tensil MD et al. A controlled trial of an internet-based intervention program for cannabis users. Cyberpsychol Behav Soc Netw 2011; 14: 673-679

[54] Tossmann HP, Kasten L. Realize it. Abschlussbericht des Bundestransfers. 2010. Im Internet: http://www.drogenbeauf tragte.de/fileadmin/dateien-dba/DrogenundSucht/Illegale_D rogen/Cannabis/Downloads/Realize_it-Transfer Abschlussbericht.pdf; Stand: 13.08.2019

[55] Hoch E, Bühringer G, Pixa A et al. CANDIS treatment program for cannabis use disorders: Findings from a randomized multisite translational trial. Drug Alcohol Dependence 2014; 134: 185-193

[56] Hoch E, Zimmermann P, Henker J et al. Modulare Therapie von Cannabisstörungen. Das CANDIS-Programm. Göttingen: Hogrefe; 2011

[57] Hoch E, Noack R, Henker J et al. Efficacy of a targeted cognitive-behavioral treatment program for cannabis use disorders (CANDIS). Eur Neuropsychopharmacol 2012; 22: 267-280 
[58] Neumann M, Bühringer $G$, Hoefler $M$ et al. Is cannabis use treatment also indicated for patients with low to moderate polysubstance use? Eur Addict Res 2018; 24: 79-87
Bibliografie

DOI https://doi.org/10.1055/a-1008-7427

Fortschr Neurol Psychiatr 2019; 87: 714-728

(c) Georg Thieme Verlag KG Stuttgart · New York ISSN 0720-4299 


\section{Punkte sammeln auf GME.thieme.de}

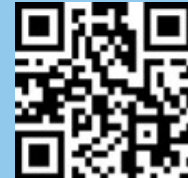

Diese Fortbildungseinheit ist in der Regel 12 Monate online für die Teilnahme verfügbar.

Den genauen Einsendeschluss finden Sie unter https://eref.thieme.de/CXDTP75.

Sollten Sie Fragen zu Online-Teilnahme haben, finden Sie unter https://cme.thieme.de/hilfe eine ausführliche Anleitung. Wir wünschen viel Erfolg beim Beantworten der Fragen!

Unter https://eref.thieme.de/CXDTP75 oder über den QR-Code kommen Sie direkt zur Startseite des Wissenstests.

VNR 2760512019156642082

\section{Frage 1}

Wie hoch ist nach Angaben des Bundeskriminalamts der durchschnittliche THC-Gehalt in Cannabisharz in Deutschland?

$\begin{array}{ll}\text { A } & 14,7 \% \\ \text { B } & 47 \% \\ \text { C } & 90,2 \% \\ \text { D } & 2,3 \% \\ \text { E } & \text { in den Produkten nicht mehr vorhanden }\end{array}$

\section{Frage 2}

Welche Aussage ist richtig? Cannabinoide entfalten ihre psychotropen Effekte vor allem über. . .

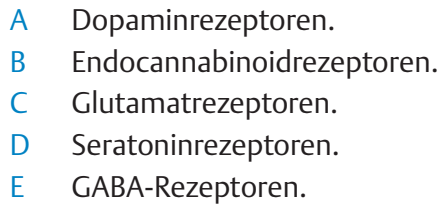

\section{Frage 3}

Wie hoch ist die 12-Monats-Prävalenz für Cannabiskonsum bei 18- bis 25-Jährigen?

$\begin{array}{ll}\text { A } & 1 \% \\ \text { B } & 2 \% \\ \text { C } & \text { ca. } 6 \% \\ \text { D } & \text { ca. } 18 \% \\ \text { E } & 25 \%\end{array}$

\section{Frage 4}

Wie hoch ist der Circaanteil aller Cannabiskonsumenten, die im Verlauf eine cannabisbezogene Störung entwickeln?

$\begin{array}{ll}\text { A } & 1 \% \\ \text { B } & 5 \% \\ \text { C } & 9 \% \\ \text { D } & 15 \% \\ \text { E } & 20 \%\end{array}$

\section{Frage 5}

Welches sind typische Cannabisentzugsbeschwerden?
A Ärger, Unruhe, Schwitzen
B Übelkeit, Durchfall und Erbrechen
C grippeähnliche Symptome
D Tränenfluss, Nase läuft
E mehr Appetit und Gewichtszunahme

\section{Frage 6}

Wie hoch ist nach aktuellen Metaanalysen bei häufigem Cannabiskonsum das Risiko, an Psychosen zu erkranken?
A nicht erhöht
B auf weniger als das 2-Fache erhöht
C auf das 3-Fache erhöht
D auf das 5-Fache erhöht
E auf das 10-Fache erhöht

\section{Frage 7}

Welche Aussage trifft zu? Somatische Folgen des Konsums von Cannabiszigaretten betreffen vor allem...
A Schilddrüse
B Haut
C Knochen
D Prostata
E Bronchialsystem

\section{Frage 8}

Welche evidenzbasierten Therapien zur Pharmakotherapie des Cannabisentzugssyndroms gibt es?
A keine
B Benzodiazepine
C Gabapentin
D niederpotente Neuroleptika
E Antidepressiva 


\section{Punkte sammeln auf CMIE.thieme.de}

Fortsetzung ...

\section{Frage 9}

Wie viele mögliche Symptome gibt es im DSM-5 insgesamt für Substanzgebrauchsstörungen?
A 4 mögliche Symptome
B 6 mögliche Symptome
C 8 mögliche Symptome
D 10 mögliche Symptome
E 11 mögliche Symptome

\section{Frage 10}

Bei wie vielen Symptomen ist die Schwelle für eine Substanzgebrauchsstörung von Cannabinoiden im DSM-5 überschritten?
A 1 mögliches Symptom
B 2 mögliche Symptome
C 3 mögliche Symptome
D 5 mögliche Symptome
E 11 mögliche Symptome 